\title{
Modélisation quasi-tridimensionnelle des écoulements dans les turbomachines hydrauliques
}

\author{
Quasi three-dimensional flows in hydraulic turbomachines
}

\author{
L. R. Eremeef et $\mathbb{R}$. Philibert
}

Société Neyrpic, Grenoble

\section{Introduction}

Aujourd'hui, comm par le passé, l'amélioration des performances hydrauliques passe par la connaissance du champ d'écoulement dans les organes de la turbomachine. C'est la raison pour laquelle NEYRPIC a développé ces demières années de nouveaux programmes de calcul qui font appel à la théorie des écoulements quasi-tridimensionnels.

La méthode consiste à modeliser mathématiquement une première approximation des conduits hydrauliques par un ensemble de filets fluides en équilibre radial (fig. 1). Ces premières données permettent de calculer l'écoulement dans la machine élémentaire constituée par la grille d'aubes tracée sur les surfaces de révolution caractérisant le filet fluide correspondant. Le calcul prend en compte l'effet dû à la variation du rayon et l'effet dû à la variation d'épaisseur $\Delta b$ de la turbomachine élémentaire. Ces résultats servent ensuite à définir une nouvelle approximation du réseau d'écoulement méridien jusqu'à convergence de la solution. La méthode fait donc appel à deux programmes couplés :

1) Calcul de l'écoulement, en général rotationnel, dans les conduits axisymétriques, l'effet de la grille d'aubes est représenté par un champ de force judicieusement choisi.

2) Calcul de l'écoulement dans une grille d'aubes tracée sur une surface de révolution de méridienne quelconque.

\section{Bases théoriques}

\subsection{Modèle physique adopté}

L'écoulement réel dans une turbomachine a un caractère tridimensionnel, instationnaire et visqueux. A l'heure actuelle il n'existe pas de méthode numérique standard, fiable et rapide, qui résolve les équations de
Navier-Stokes dans leur ensemble. Ceci nous conduit à introduire des approximations et à négliger certains phénomènes. Nous supposerons tout d'abord que l'écoulement peut se partager en un écoulement principal sans frottement et un écoulement pariétal avec frotte-
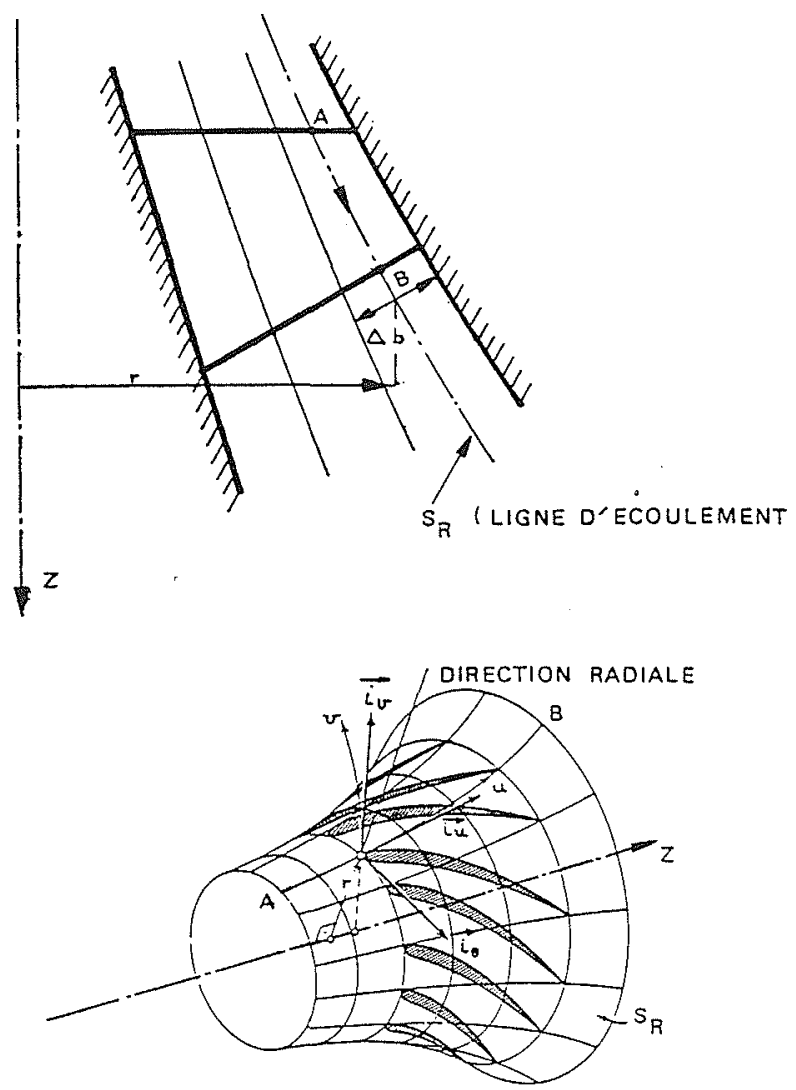

Figure 1 - Ecoulement quasi-tri-dimensionnel sur une surface axisy métrique 
ment du type couche limite. Nous nous intéresserons ici à l'écoulement d'un fluide parfait, incompressible et stationnaire, par rapport à un repère lié aux aubages (écoulement permanent dans le repère relatif). Nous introduirons toutefois dans les équations de la mécanique un champ de force $\vec{f}$ simulant les pertes.

\subsection{Equation du mouvement absolu en projection sur les axes mobiles [1]}

Elle s'écrit sous forme vectorielle :

$$
\overrightarrow{\operatorname{grad}}\left[\frac{p}{\rho}+g z+\frac{V^{2}}{2}-\vec{U} \cdot \vec{V}\right]=\vec{w}_{\wedge} \overrightarrow{\operatorname{rot}} V+\vec{f}
$$

dans laquelle :

$\frac{p}{\rho}+g z+\frac{V^{2}}{2}$ est l'expression de l'énergie absolue $E_{a b s}$ $\frac{p}{\rho}+g z+\frac{V^{2}}{2}-\vec{U} \cdot \vec{V}$ est l'expression de l'énergie relative $E_{\text {rel }}$ qui s'écrit aussi $\frac{p}{\rho}+g z+\left(W^{2}-U^{2}\right) / 2$

Nous remarquerons, quand l'absence de champ $\vec{f}$, l'énergie relative se conserve le long de la trajectoire d'une particule fluide. En effet, multiplions scalairement les deux membres de l'équation (1) par le vecteur $\vec{W}$, on obtient :

$\frac{D E_{\mathrm{rel}}}{D t}=\vec{W} \cdot \overrightarrow{\operatorname{grad}} E_{\mathrm{rel}}=\vec{W} \cdot\left(\vec{W}_{\wedge} \overrightarrow{\mathrm{rot}} V\right)$

$$
=\overrightarrow{\operatorname{rot}} V \cdot\left(\vec{w}_{\wedge} \vec{W}\right)=0
$$

\subsection{Equation de continuité}

$$
\frac{\partial\left(r V_{r}\right)}{\partial r}+\frac{\partial V_{\theta}}{\partial \theta}+\frac{\partial\left(r V_{z}\right)}{\partial z}=0
$$

\subsection{Conditions aux limites}

Ce sont essentiellement :

- la donnée des vitesses normales à la surface limitant le volume où l'on étudie l'écoulement et

la condition de glissement sur les aubes, la ceinture et le plafond.

Des conditions supplémentaires doivent être satisfaites Citons par exemple :

- la périodicité de l'écoulement et

- la condition de Kutta Joukovski.

\section{Transformation des équations tridimension- nelles}

\subsection{Le modèle de Chung-Hua Wu[2]}

L'écoulement n'est stationnaire dans le repère relatif que si les conditions d'entrée du mouvement absolu sont axisymétriques [3]. On peut montrer également que la présence d'un aubage fixe ou mobile détruit cette axisymétrie.

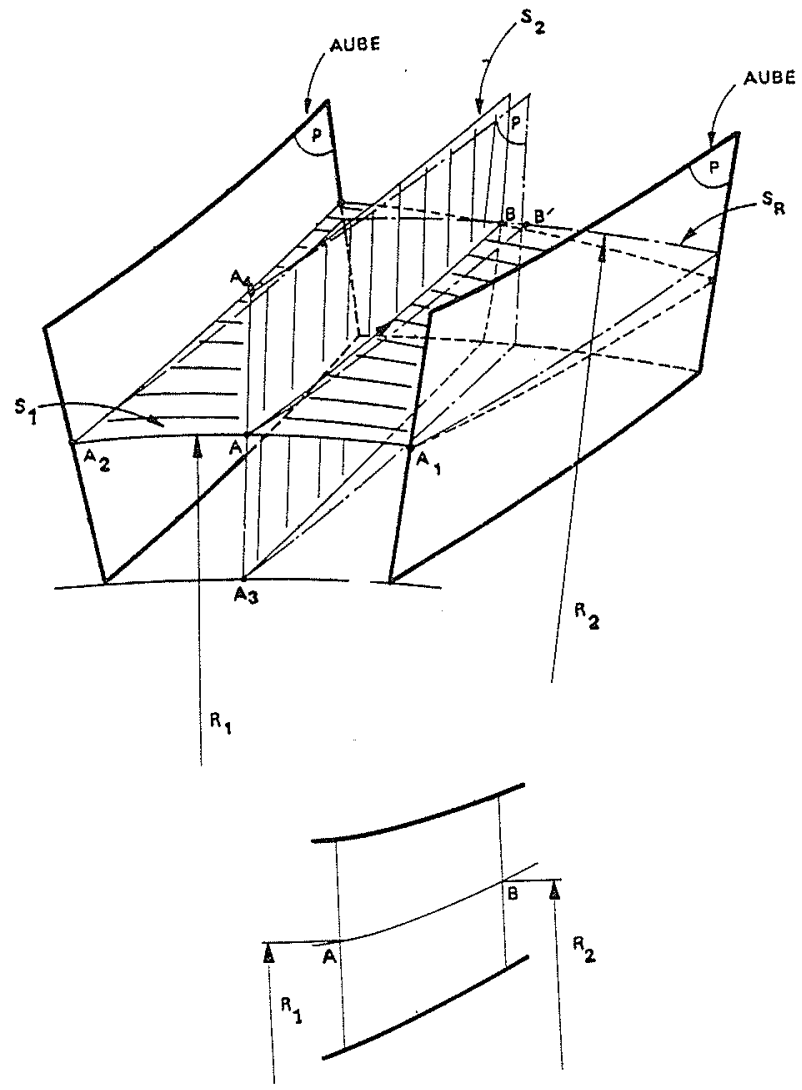

Figure 2 - Ecoulement tri-dimensionnel dans un canal inter-aube

C'est la raison pour laquelle l'écoulement à travers une grille d'aubes tridimensionnelle ne peut pas s'effectuer sur des surfaces de révolution. La figure 2 schématise une telle situation dans un canal inter-aube pour lequel nous avons adopté, pour des raisons pratiques, un aubage infiniment mince et une arête d'entrée et de sortie purement radiale. La surface $S_{1}$ est le lieu des particules fluides entrant au rayon $R_{1}$ tandis que $S_{2}$ est celui des particules entrant suivant $A_{3} A_{4}$. Nous avons représenté sur cette figure la surface de révolution $S_{R}$ de méridienne $\mathrm{AB}$ qui est l'intersection des surfaces $S_{1}$ et $S_{2}$.

On voit nettement qu'une particule fluide entrant au même rayon et à la même côte en $A_{1}$ ou $A_{2}$ n'a pas le mëme trajet sur l'intrados ou l'extrados. De mème la trajectoire $A B$ d une particule fluide sur $S_{2}$ est différente de la méridienne $A B^{\prime}$ intersection de $S_{R}$ et de la pale.

Pour chaque surface, les coordonnées et leurs différentielles sont liées par:

$$
\begin{gathered}
S(r, \theta, z)=0 \\
n_{r} d_{r}+n_{\theta} r d \theta+n_{z} d z=0
\end{gathered}
$$

$\vec{n}$ est le vecteur unitaire normal à $S$, perpendiculaire à $\vec{W}$. La condition de glissement s'écrit :

$$
\vec{n} \cdot \vec{W}=0
$$

La méthode de $W u$ consiste à éliminer l'une des trois coordonnées $r, \theta, z$, dans les équations (1) et (2) en faisant appel à la notion de dérivée suivant une surface de courant (annexe II). 
$W u$ introduit ensuite une fonction de courant et obtient une équation principale en $\psi$, du type de Poisson, dont la solution décrit l'écoulement sur les surfaces $S_{1}$ ou $S_{2}$.

Le schéma de calcul s'établit ainsi :

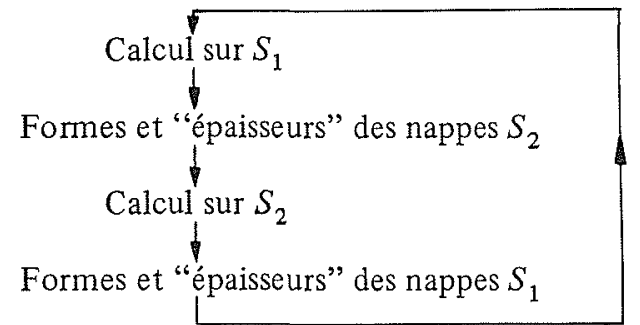

Dans la pratique la convergence du schéma est longue et délicate car $S_{1}$ et $S_{2}$ sont des surfaces vrillées quelconques. C'est la raison pour laquelle on suppose généralement que les surfaces $S_{1}$ sont presque des surfaces de révolution et que les surfaces $S_{2}$ sont voisines de la surface squelette de la pale.

Cette hypothèse est d'autant plus vraie que le nombre d'aubes est élevé ou que l'envergure de la pale est petite vis-à-vis du rayon. On considère donc en première approximation que l'écoulement se développe sur des surfaces $S_{1}$ axisymétriques et qu'il suffit d'une seule surface $S_{2}$ pour établir la forme et les "épaisseurs" des nappes d'écoulement $S_{1}$. L'écoulement sur $S_{1}$ sera considéré comme bi-dimensionnel.

\subsection{Ecoulement sur $S_{2}$ (ou écoulement méridien)}

\subsubsection{Continuité}

Utilisons les relations (II c) annexe II pour transformer l'équation (2).

Nous avons

$\frac{\overline{\partial\left(r V_{r}\right)}}{\partial r}+\frac{\overline{\partial\left(r V_{z}\right)}}{\partial z}=-\frac{1}{n_{\theta}}\left[n_{r} \frac{\partial V_{r}}{\partial \theta}+n_{\theta} \frac{\partial V_{\theta}}{\partial \theta}+n_{z} \frac{\partial V_{z}}{\partial \theta}\right]$ mais $\vec{n}$ est indépendant de $\theta$ et compte-tenu de (Ia) nous pouvons écrire :

$$
\left.\frac{\overline{\partial\left(r V_{r}\right)}}{\partial r}+\frac{\overline{\partial\left(r V_{z}\right)}}{\partial z}=-\frac{1}{n_{0}} \frac{\partial}{\partial \theta} \overrightarrow{[n} \cdot \vec{w}\right]=0
$$

Pour un écoulement axisymétrique l'équation de continuité est indépendante de $S_{2}$.

\subsubsection{Mécanique}

Plaçons nous dans le repère $(v, \theta, u)$ (fig. 3), l'équation (1) explicitée dans ce repère local s'écrit :

$$
\begin{aligned}
& \frac{\partial E_{\mathrm{rel}}}{\partial v}=W_{\theta} \omega_{u}-w_{u} \omega_{\theta}+f_{v} \\
& \frac{1}{r} \frac{\partial E_{\mathrm{rel}}}{\partial \theta}=w_{u} \omega_{v}-w_{v} \omega_{u}+f_{\theta} \\
& \frac{\partial E_{\mathrm{rel}}}{\partial u}=w_{v} \omega_{\theta}-W_{\theta} \omega_{v}+f_{u}
\end{aligned}
$$

On peut montrer, à l'aide des relations (II') que ces équations deviennent:

$$
\begin{gathered}
\frac{\overline{\partial E_{\mathrm{rel}}}}{\partial v}=\frac{W_{\theta}}{r} \frac{\overline{\partial r V_{\theta}}}{\partial v}-W_{u} \bar{\omega}_{\theta}+F_{v}+f_{v} \\
0=\frac{-1}{r}\left[W_{u} \frac{\overline{\partial r V_{\theta}}}{\partial u}+W_{v} \frac{\overline{\partial r V_{\theta}}}{\partial v}\right]+F_{\theta}+f_{\theta} \\
\frac{\overline{\partial E_{\mathrm{rel}}}}{\partial u}=W_{v} \bar{\omega}_{\theta}+\frac{W_{\theta}}{r} \frac{\overline{\partial r V_{\theta}}}{\partial u}+F_{u}+f_{u}
\end{gathered}
$$

dans lesquelles on a posé :

$$
\begin{aligned}
\bar{\omega}_{\theta} & =\frac{\overline{\partial V_{v}}}{\partial u}-\frac{\overline{\partial V_{u}}}{\partial v}+V_{v} \frac{\overline{\partial \gamma}}{\partial v}+V_{u} \frac{\overline{\partial \gamma}}{\partial u} \\
\text { et } \quad \vec{F} & =-\frac{1}{r} \frac{\vec{n}}{n_{\theta}} \frac{1}{\rho} \frac{\partial p^{*}}{\partial \theta}
\end{aligned}
$$

$\vec{F}$ apparait comme un vecteur ayant la dimension d'une force par unité de masse fluide. Il permet de prendre en compte, au niveau de l'écoulement sur $S_{2}$, l'action du rotor ou du stator sur le fluide.

Multiplions maintenant l'équation (9) par $W_{v}$, (10) par $W_{\theta}$ et (11) par $W_{u}$, additionnons et si nous tenons compte du fait que l'écoulement est bidimensionnel sur $S_{1}\left(W_{v}=0\right)$, nous obtenons :

$$
W_{u} \frac{\overline{\partial E_{\mathrm{rel}}}}{\partial u}=w_{u} f_{u}+w_{\theta} f_{\theta}
$$

Faisons l'hypothèse suivant laquelle $\vec{f}$ est colinéaire à $\vec{W}$, nous avons les relations :

$$
\begin{gathered}
f_{v}=0 \\
\frac{f_{u}}{W_{u}}=\frac{f_{\theta}}{W_{\theta}}=\frac{f}{W}
\end{gathered}
$$

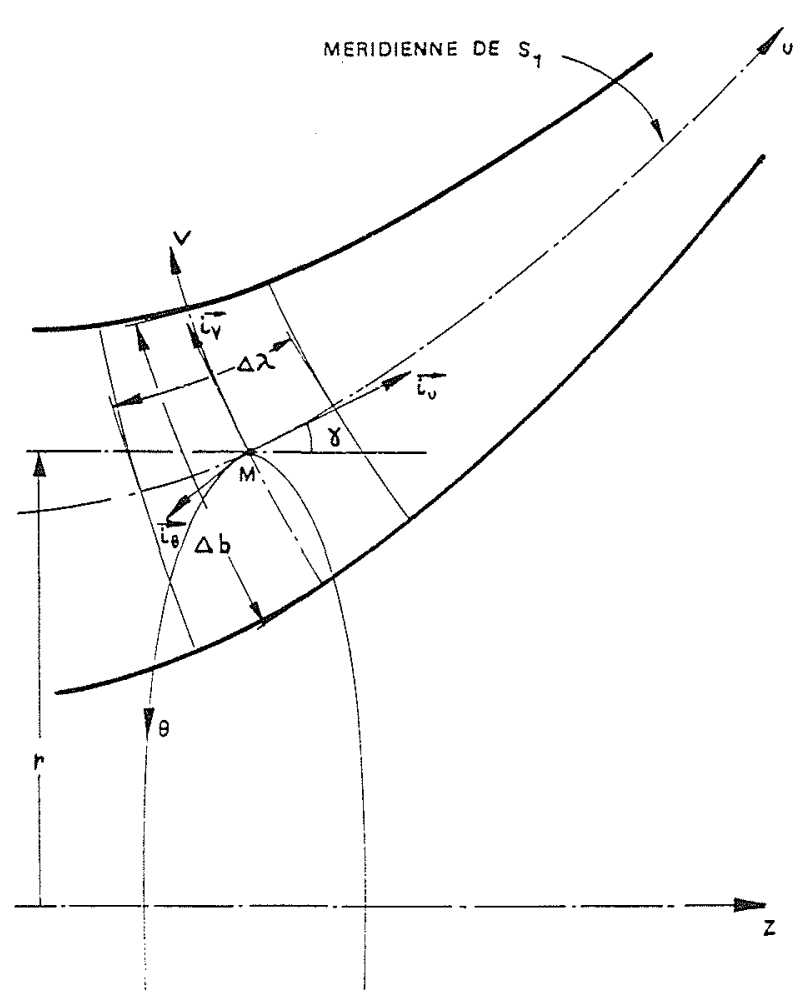

Figure 3 - Système de coordonnées curvilignes orthogonales 
Nous pouvons facilement établir à partir de (12) et (13) l'expression de $f_{u}$ et $f_{\theta}$. On trouve :

$$
\begin{aligned}
& f_{u}=\left(\frac{W_{u}}{W}\right)^{2} \frac{\overline{\partial E_{\mathrm{rel}}}}{\partial u} \\
& f_{\theta}=\frac{W_{u} W_{\theta}}{W^{2}} \frac{\overline{\partial E_{\mathrm{rel}}}}{\partial u}
\end{aligned}
$$

Reportons ces valeurs dans (10) et (11), le système d'équations (9), (10), (11) se réduit à :

$$
\begin{aligned}
\check{\bar{\omega}}_{\theta} & =\frac{F_{v}}{W_{u}}-\frac{1}{W_{u}} \frac{\overline{\partial E_{\mathrm{rel}}}}{\partial v}+\frac{1}{r} \frac{W_{\theta}}{W_{u} \frac{\overline{\partial r V_{\theta}}}{\partial v}} \\
F_{\theta} & =\frac{W_{u}}{r} \frac{\overline{\partial r V_{\theta}}}{\partial u}-\frac{W_{u} W_{\theta}}{W^{2}} \frac{\overline{\partial E_{\mathrm{rel}}}}{\partial u} \\
F_{u} & =\frac{\overline{\partial E_{\mathrm{rel}}}}{\partial u}-\frac{W_{\theta}}{r} \frac{\overline{\partial r V_{\theta}}}{\partial u}-\left(\frac{W_{u}}{W}\right)^{2} \frac{\overline{\partial E_{\mathrm{rel}}}}{\partial u}
\end{aligned}
$$

Nous voyons que les composantes $F_{u}$ et $F_{\theta}$ s'expriment également en fonction du gradient de $r V_{\theta}$ et de $E_{\text {rel }}$ pris dans la direction $u$. Il n'est pas difficile d'exprimer la troisième composante $F_{v}$ en fonction des mêmes gradients puisque $\vec{F}$ et $\vec{n}$ sont colinéaires.

$$
F_{v}=\frac{n_{v}}{n_{\theta}} F_{\theta} \text { ou bien } F_{v}=\frac{n_{v}}{n_{u}} F_{u}
$$

les deux expressions sont équivalentes et nous obtenons :

$$
F_{v}=\frac{n_{v}}{n_{\theta}} \frac{W_{u}}{r} \frac{\overline{\partial r V_{\theta}}}{\partial u}-\frac{n_{v}}{n_{\theta}} \frac{W_{u} W_{\theta}}{W^{2}} \frac{\overline{\partial E_{\mathrm{rel}}}}{\partial u}
$$

En définitive l'équation principale (14) associée à l'équation (5) définissent les équations de l'écoulement méridien qui est un problème bi-dimensionnel dans le plan $(r, z)$. Nous pourrons traiter les dérivées surlignées comme des dérivées ordinaires.

Remarque : l'équation de continuité (5) n'est valable que pour un aubage infiniment mince et doit être remplacée par l'équation :

$$
\frac{\partial\left(\tau r V_{r}\right)}{\partial r}+\frac{\partial\left(\tau r V_{2}\right)}{\partial z}=0
$$

pour un aubage d'épaisseur finie

Ici $\tau=1-\frac{N \Delta \theta(r, z)}{2 \pi}$ est le coefficient d'obstruction dû à la présence de l'aubage.

\subsubsection{Equation résolvante de l'écoulement méridien}

Introduisons la fonction de courant $\psi$ définit par :

$$
\begin{aligned}
\tau r V_{r} & =\frac{\partial \psi}{\partial z} \\
\tau r V_{z} & =\frac{-\partial \psi}{\partial r}
\end{aligned}
$$

L'équation (17) est automatiquement vérifiée et (14) peut s'écrire :

$$
\omega_{\theta}=\frac{\partial V_{r}}{\partial z}-\frac{\partial V_{z}}{\partial r}=\frac{-1}{W_{u}} \cdot \frac{\partial E_{\mathrm{rel}}}{\partial v}+\frac{1}{r} \frac{W_{\theta}}{W_{u}} \frac{\partial r V_{\theta}}{\partial v}+\frac{n_{v}}{n_{\theta}}\left[\frac{1}{r} \frac{\partial r V_{\theta}}{\partial u}-\frac{W_{\theta}}{W^{2}} \frac{\partial E_{\mathrm{rel}}}{\partial u}\right]
$$

avec :

$$
\frac{\partial V_{r}}{\partial z}-\frac{\partial V_{z}}{\partial r}=\frac{1}{\tau r}\left[\frac{\partial^{2} \psi}{\partial r^{2}}+\frac{\partial^{2} \psi}{\partial z^{2}}\right]+\frac{W_{u}}{\tau} \frac{\partial \tau}{\partial v}+\frac{V_{z}}{r}
$$

d'où l'équation finale :

$$
\begin{aligned}
\frac{\partial^{2} \psi}{\partial r^{2}} & +\frac{\partial^{2} \psi}{\partial z^{2}}=\tau\left\{-\frac{r}{W_{u}} \frac{\partial E_{\mathrm{rel}}}{\partial v}+\frac{W_{\theta}}{W_{u}} \frac{\partial r V_{\theta}}{\partial v}+\right. \\
& \left.+\frac{n_{v}}{n_{\theta}}\left[\frac{\partial r V_{\theta}}{\partial u}-\frac{r W_{\theta}}{W^{2}} \frac{\partial E_{\mathrm{rel}}}{\partial u}\right]-V_{z}\right\}-r W_{u} \frac{\partial \tau}{\partial v}(18)
\end{aligned}
$$

\subsection{Ecoulement sur $S_{I}$ (ou écoulement aube à aube}

\subsubsection{Continuité}

L'équation de continuité sur $S_{1}$ est obtenue à partir de (If) dans laquelle on fait $V_{v}=0$ :

$$
\frac{\partial\left[r \Delta b V_{u}\right]}{\partial u}+\frac{1}{r} \frac{\partial\left[r \Delta b V_{\theta}\right]}{\partial \theta}=0
$$

$\Delta b(u)$ représente la variation d'épaisseur des nappes $S_{1}$. L'application des formules (II d) dans l'équation précédente est triviale et ne modifie pas sa forme définitive.

\subsubsection{Mécanique}

Nous supposerons dans ce qui suit que $\vec{f}=0$. Exprimons (6) (7) et (8) en utilisant (II d) nous avons :

$$
\begin{gathered}
\frac{\overline{\partial E_{\mathrm{rel}}}}{\partial u}=-W_{\theta} \bar{\omega}_{v} \\
\frac{1}{r} \frac{\overline{\partial E_{\mathrm{rel}}}}{\partial \theta}=W_{u} \bar{\omega}_{v} \\
\frac{1}{\rho} \frac{\partial p^{*}}{\partial v}=\left(U+W_{\theta}\right)\left(\frac{\partial U}{\partial v}+\frac{W_{\theta}}{r} \frac{\partial r}{\partial v}\right)-w_{u}^{2} \frac{\partial \gamma}{\partial u}
\end{gathered}
$$

La dernière équation est l'équation de l'équilibre radial du filet fluide dans le plan $(r, z)$. Elle ne nous aide en rien pour l'étude de l'écoulement sur $S_{1}$. On vérifie que $W_{u} \frac{\overline{\partial E_{\mathrm{rel}}}}{\partial u}+W_{\theta} \frac{1}{r} \frac{\overline{\partial E_{\mathrm{rel}}}}{\partial \theta}=0$ sur $S_{1}$ quelque soit $\bar{\omega}_{v}$.

Mais puisque l'écoulement à l'amont du rotor ou du stator est axisymétrique $\frac{\partial E_{\mathrm{rel}}}{\partial \theta}=0$ et $\bar{\omega}_{v}=0$.

En vertu du théorème de Kelvin, on pourra dire que $\omega_{v}$ est nul partout sur $S_{1}$ et l'équation de la mécanique se réduit à :

$$
\frac{\partial V_{u}}{\partial \theta}-\frac{\partial\left(r V_{\theta}\right)}{\partial u}=0
$$

\subsubsection{Equation résolvante}

L'étude de l'écoulement se fait dans un plan $(x, y)$ au moyen de la transformation: 


$$
\begin{gathered}
x=k\left(-\frac{1}{2}+\frac{\int_{0}^{u} \frac{d u}{r}}{\int_{0}^{u_{0}} \frac{d u}{r}}\right) \\
y=k \frac{\theta}{\int_{0}^{u_{0}} \frac{d u}{r}}
\end{gathered}
$$

où $k$ peut se considérer comme un facteur d'échelle arbitraire et $u_{0}$ représente l'abscisse de sortie de l'aubage (l'origine des abscisses étant pris à l'entrée de ce dernier).

La transformations précédente possède la propriété des transformations conformes (conservation des angles mais pas des longueurs). En particulier la circulation se conserve dans cette transformation.

Les vitesses dans le plan $(x, y)$ sont liées aux vitesses de l'espace réel par la relation

$$
V^{*}=\frac{r \int_{0}^{u_{0}} \frac{d u}{r}}{k} V
$$

Les équations (19) et (23) s'expriment dans ce plan sous la forme

$$
\begin{gathered}
\frac{\partial W_{y}^{*}}{\partial x}-\frac{\partial W_{x}^{*}}{\partial y}=-\frac{d U_{y}^{*}}{d x} \\
\frac{\partial\left(\Delta b W_{x}^{*}\right)}{\partial x}+\frac{\partial\left(\Delta b W_{y}^{*}\right)}{\partial y}=0
\end{gathered}
$$

Pour l'écoulement relatif, on pose :

$$
\begin{aligned}
& W_{x}^{*}=-\frac{1}{\Delta b} \frac{\partial \psi^{*}}{\partial y} \\
& \mathrm{~W}_{y}^{*}=\frac{1}{\Delta b} \frac{\partial \psi^{*}}{\partial x}
\end{aligned}
$$

où $\psi *$ est une forme de courant.

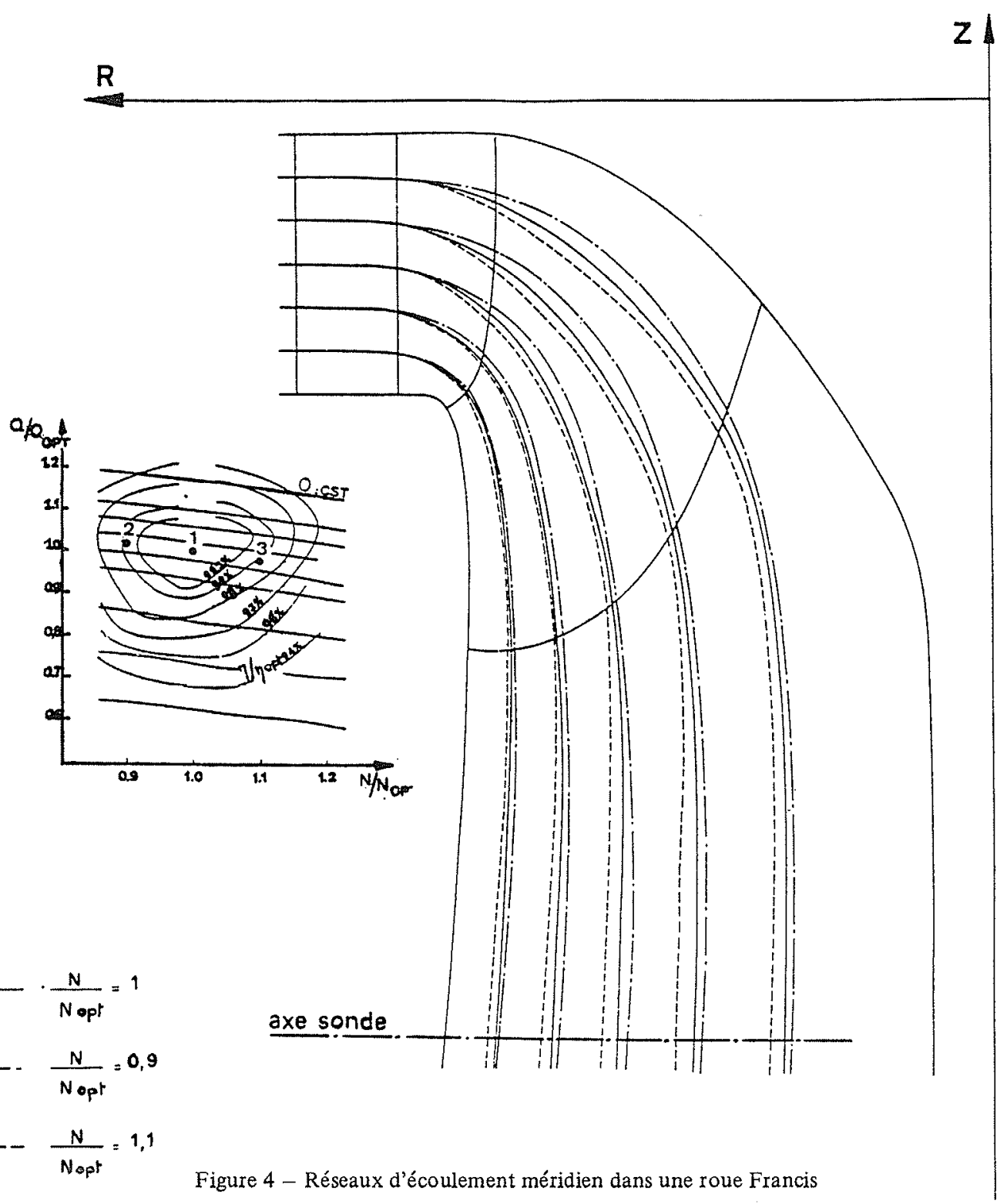




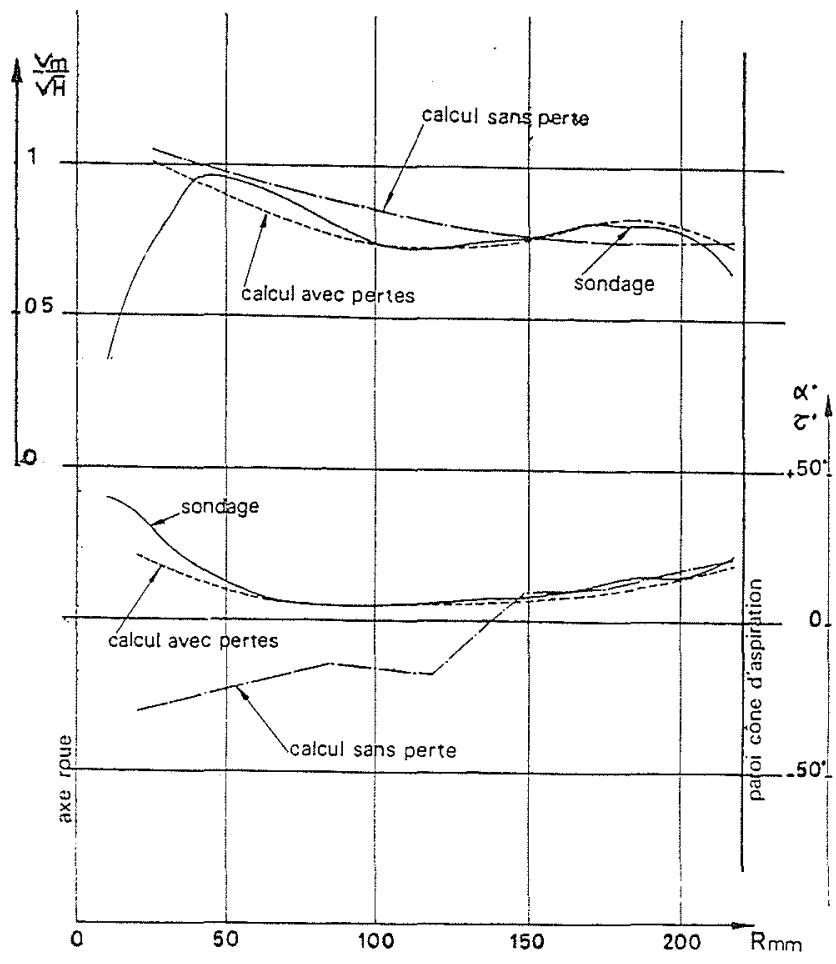

Figure 5 - Comparaison calcul-sondage sous une roue Francis

L'équation (24) devient :

$$
\frac{\partial^{2} \psi^{*}}{\partial x^{2}}+\frac{\partial^{2} \psi^{*}}{\partial y^{2}}=\frac{d \Delta b}{d x} W_{y}^{*}-\Delta b \frac{d U_{y}^{*}}{d x}
$$

\subsection{Technique itérative de résolution}

L'objet principal de l'étude est la détermination du champ des vitesses dans le conduit méridien ou le long du contour des aubages sur $S_{1}$. L'examen des relations (18) et (26) montre que ces deux équations sont de même nature et peuvent se résoudre par le même type d'algorithme. Nous avons choisi à NEYRPIC la méthode intégrale pour laquelle nous possédons depuis longtemps une grande expétience de son utilisation. Le développement complet d'une équation du type de Poisson par la méthode précédente sortirait du cadre de cet article. Les personnes intéressées pourront toujours consulter la bibliographie que l'on trouvera à la fin de ce bref rappel de la théorie quasi-tridimensionnelle [4] [5] $[6][7][8]$.

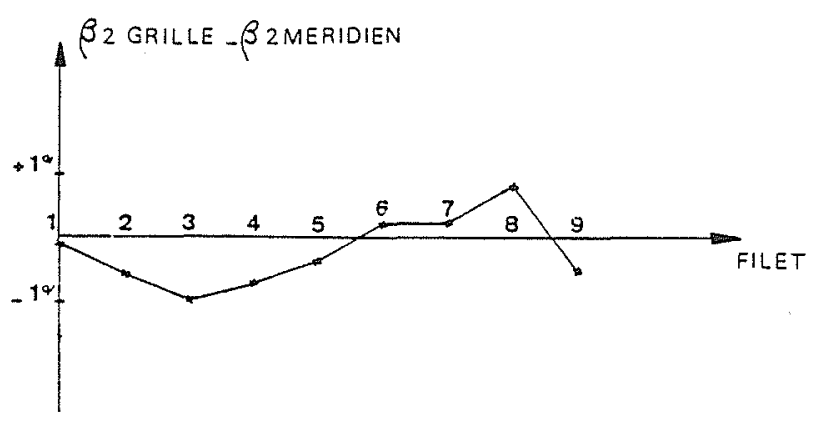

Figure 6 - Ecarts d'angles obtenus à la sortie roue d'une turbine pompe par le programme méridien et le programme aube à aube
Nous dirons simplement que les relations intégrales de Green permettent de définir la solution des équations différentielles (18) et (26).

On peut montrer que la solution du problème s'obtient par voie itérative à l'aide d'un système de deux équations intégrales de Fredholm de deuxième espèce. Elles permettent respectivement de déterminer soit les vitesses le long du contour du domaine à étudier, soit les vitesses au sein même du domaine.

L'étude de chaque écoulement a fait l'objet d'un programme séparé et par suite de la convergence rapide du procédé, le deuxième ou troisième pas d'itération foumit déjà une solution dont la précision répond parfaitement aux exigences de la pratique.

L'équation (18) montre que le calcul de l'écoulement méridien nécessite la connaissance de la répartition de $r V_{\theta}$ dans tout le domaine intérieur au contour méridien étudié.

Cette répartition est le résultat du calcul en grille d'aubes. De même le calcul en grille d'aubes nécessite la connaissance de la surface $S_{1}$ axisymétrique et de la loi d'épaisseur $\Delta b(u)$. Ces données sont le résultat du calcul méridien.

On effectue alors une itération générale entre le calcul des deux écoulements qui est arrêté lorsqu'on estime que deux écoulements successifs sont suffisamment voisins.

\section{Exemples d'application}

\subsection{Roue Francis}

Les calculs ont été menés à l'ouverture passant par le coeur de la colline et pour trois valeurs de $n_{11}$ dont le $n_{11}$ du coeur (fig. 4).

Les résultats font l'objet des figures 4 et 5 . L'évolution du réseau d'écoulement pour les trois points de fonctionnement est représentée sur la figure 4 . On constate que le réseau se déplace vers la ceinture quand le $n_{11}$ augmente (donc quand la chute diminue).

La distribution des pressions sur l'intrados et l'extrados a fait l'objet d'un examen détaillé : référence [9]. Nous en retiendrons simplement que le calcul a fait apparaître une zone de pression négative contre la ceinture dans la zone des fortes courbures de l'aube en accord avec les observations visuelles.

Un essai sonde a été effectué sous la roue pour le point de fonctionnement du coeur. Le résultat du calcul a été reporté sur la figure 5 sous la forme des courbes $V_{m}(r)$ et $\alpha(r)$, et comme le modèle mathématique actuel peut tenir compte de pertes éventuelles filet par filet, nous les avons introduites dans le calcul de vérification.

On peut observer une bonne concordance avec les courbes expérimentales.

\subsection{Roue de turbine-pompe}

Les calculs sont effectués sur un modèle de turbinepompe de faible vitesse spécifique au point de rendement optimum en fonctionnement turbine. Les écarts 


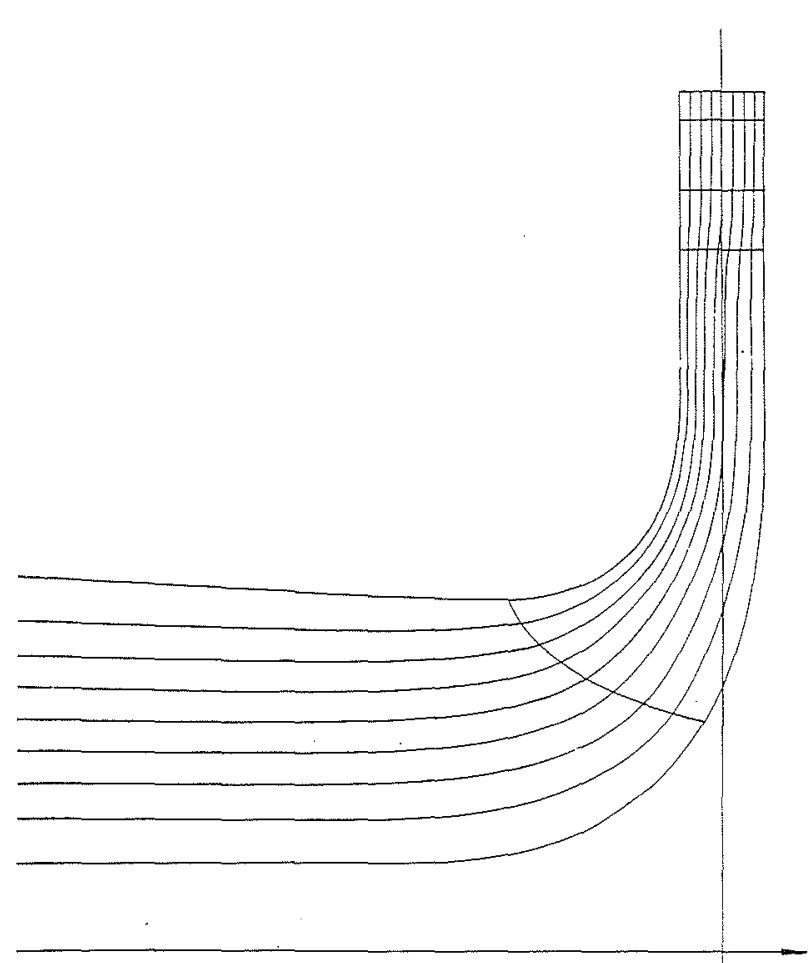

Figure 7 - Réseau d'écoulement dans une roue de turbinepompe

d'angles obtenus en fin d'itération à la sortie roue par les deux programmes d'écoulement ont été reportés sur la figure 6 . La figure 7 représente le réseau d'écoulement méridien correspondant.

Un sondage sous la roue à ce point de fonctionnement permet de vérifier les résultats de calcul. Les valeurs mesurées et calculées de la vitesse méridienne et de l'angle $\alpha$ ont été portées sur la figure 8 en fonction de $\psi$. Les écarts observés sur les répartitions de $\alpha$ au voisinage de l'axe machine proviennent de ce que le programme ne peut pas prendre en compte les rayons nuls. Remarquons cependant que le calcul conserve l'allure générale de l'évolution de $\alpha$. On peut noter, en outre, une bonne concordance entre les résultats expérimentaux et numériques en dehors de cette zone particulière.

\section{Conclusion}

Il semble à l'heure actuelle que le modèle avec pertes représente bien l'écoulement sous la roue. Il peut donc

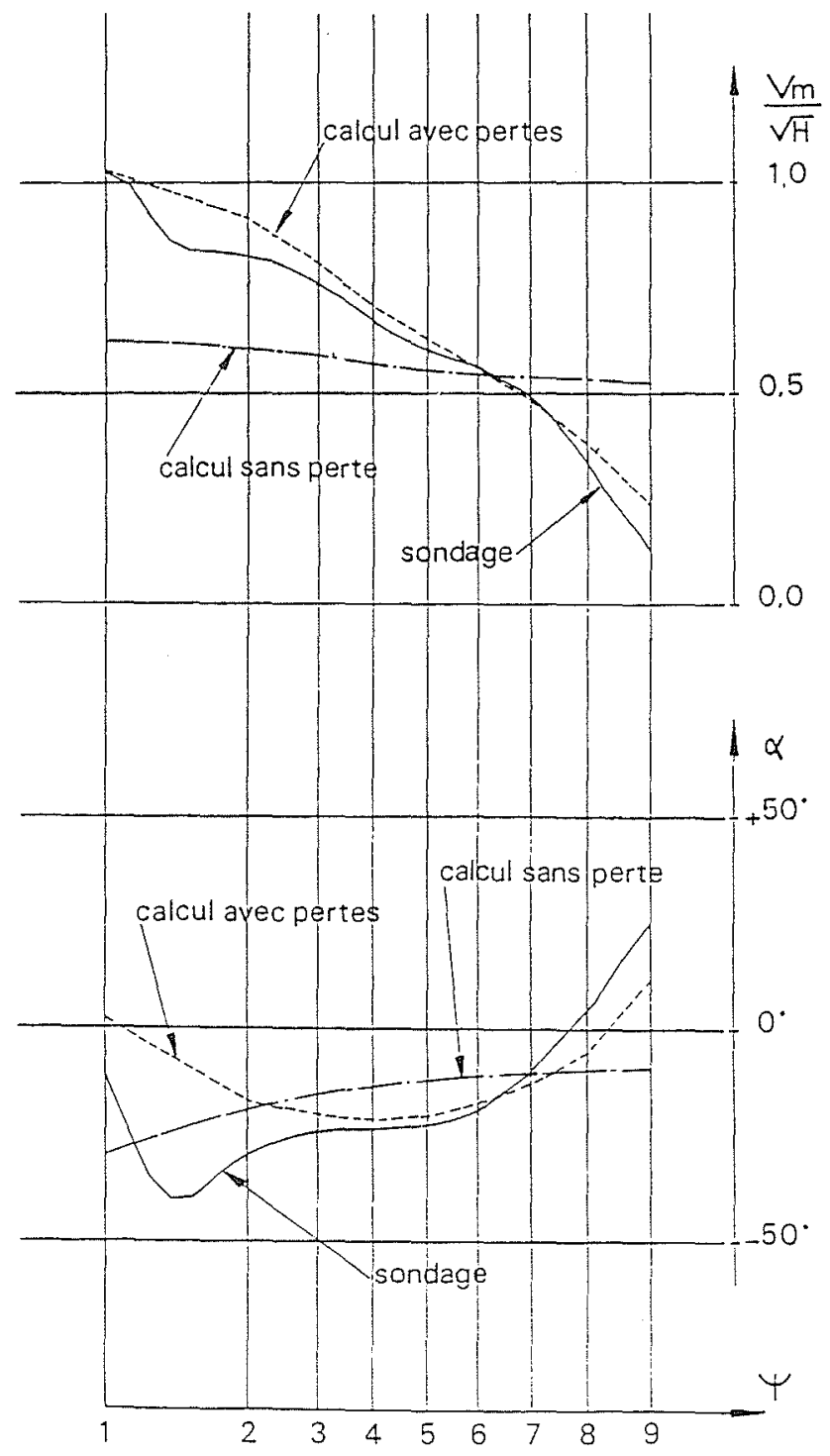

Figure 8-Comparaison calcul-sondage sous une roue de turbinepompe.

être très utile pour l'amélioration des tracés existants si nous disposons d'informations suffisantes. Il peut aussi beaucoup nous aider dans sa version fluide parfait pour la conception d'une roue nouvelle si nous savons dégager les critères d'un bon tracé.

\section{Annexe I}

\section{Notations :}

$\overrightarrow{i_{v}}, \overrightarrow{i_{\theta}}, \overrightarrow{i_{u}}$ trièdre du repère toumant lié au point $\mathrm{M}$ (fig. 3)

$\gamma$ angle du filet méridien avec l'axe machine

$N$ nombre d'aubes
$\overrightarrow{\vec{V}} \quad$ vitesse absolue $\left(V_{r}, V_{\theta}, V_{z}\right)$ ou $\left(O, V_{\theta}, V_{u}\right)$
$\vec{W} \quad$ vitesse relative $\left(W_{r}, W_{\theta}, W_{z}\right)$ ou $\left(O, W_{\theta}, W_{u}\right)$
$\vec{U} \quad$ vitesse d'entrainement $(O, U, O)$
$\vec{\Omega}=$ rot $\vec{V},\left(\omega_{r}, \omega_{\theta}, \omega_{z}\right)$ ou $\left(\omega_{v}, \omega_{\theta}, \omega_{u}\right)$
$V^{*}$ vitesse absolue dans le plan grille $\left(V_{x}^{*}, V_{y}^{*}\right)$
$W^{*}$ vitesse relative dans le plan grille $\left(W_{x}^{*}, W_{y}^{*}\right)$
$U^{*}$ vitesse d'entrainement dans le plan grille $\left(O, U_{y}^{*}\right)$ 
Relations utiles:

$$
\begin{aligned}
& V_{r}=W_{r} ; V_{z}=w_{z} ; V_{u}=W_{u} ; V_{v}=w_{v}=0 \\
& V_{\theta}=W_{\theta}+U \\
& \overrightarrow{\operatorname{rot} \vec{V}_{(r, \theta, z)}} \mid \begin{array}{ll}
\omega_{r}=\frac{1}{r}\left[\frac{\partial V_{z}}{\partial \theta}-\frac{\partial r V_{\theta}}{\partial z}\right] & \\
\omega_{\theta}=\frac{\partial V_{r}}{\partial z}-\frac{\partial V_{z}}{\partial r} & \text { (Ib) }
\end{array} \\
& \omega_{z}=\frac{1}{r}\left[\frac{\partial r V_{\theta}}{\partial r}-\frac{\partial V_{r}}{\partial \theta}\right]
\end{aligned}
$$

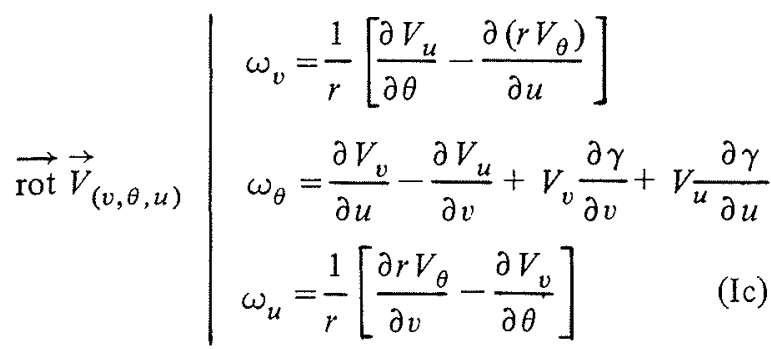

$$
\begin{aligned}
\operatorname{div} \vec{V}_{(r, \theta, z)}=\frac{\partial\left(r V_{r}\right)}{\partial r}+\frac{\partial V_{\theta}}{\partial \theta} & +\frac{\partial\left(r V_{z}\right)}{\partial z} \\
\operatorname{div} \vec{V}_{(v, \theta, u)}=\frac{1}{r} \frac{\partial r V_{u}}{\partial u}+\frac{1}{r} \frac{\partial r V_{v}}{\partial \theta} & +\frac{1}{r} \frac{\partial V_{\theta}}{\partial \theta} \\
& +V_{u} \frac{\partial \gamma}{\partial v}-V_{v} \frac{\partial \gamma}{\partial u}
\end{aligned}
$$

On peut montrer que [3] :

$$
\begin{gathered}
\frac{\partial \gamma}{\partial v}=\frac{1}{\Delta b} \frac{\partial \Delta b}{\partial u} \\
-\frac{\partial \gamma}{\partial u}=\frac{1}{\Delta \lambda} \frac{\partial \Delta \lambda}{\partial v}
\end{gathered}
$$

d'où

$$
\begin{aligned}
\operatorname{div} \vec{V}_{(v, \theta, u)}=\Delta \lambda \frac{\partial\left[r \Delta b V_{u}\right]}{\partial u}+ & \Delta b \frac{\partial\left[r \Delta \lambda V_{v}\right]}{\partial v} \\
& +\frac{\Delta b \Delta \lambda}{r} \frac{\partial r V_{\theta}}{\partial \theta}
\end{aligned}
$$

\section{Annexe II}

Définition des dérivées surlignées ou dérivées suivant une surface de courant

Soit $S(r, \theta, z)$ l'équation de la surface de courant, nous avons :

$$
\begin{aligned}
d S & =\frac{\partial S}{\partial r} d r+\frac{\partial S}{\partial z} d z+\frac{1}{r} \frac{\partial S}{\partial \theta}(r d \theta) \\
& =n_{r} d r+n_{\theta} r d \theta+n_{z} d z=0
\end{aligned}
$$

où $\vec{n}$ est le vecteur unitaire normal à $S$.

Les dérivées surlignées d'une fonction scalaire $f(r, \theta$, $z)$ se définiront comme suit :

$$
d f=\frac{\partial f}{\partial r} d r+\frac{1}{r} \frac{\partial f}{\partial \theta}(r d \theta)+\frac{\partial f}{\partial z} d z=\frac{\overline{\partial f}}{d r} d r+\frac{\overline{\partial f}}{\partial z} d z
$$

pour l'écoulement sur $S_{2}$, mais :

$$
r d \theta=-\frac{n_{r}}{n_{\theta}} d r-\frac{n_{z}}{n_{\theta}} d z
$$

En remplaçant dans (IIb) $r d \theta$ par sa valeur et en identifiant terme a terme nous obtenons:

$$
\begin{aligned}
& \overline{\frac{\partial f}{\partial r}}=\frac{\partial f}{\partial r}-\frac{1}{r} \frac{n_{r}}{n_{\theta}} \frac{\partial f}{\partial \theta} \\
& \overline{\frac{\partial f}{\partial z}}=\frac{\partial f}{\partial z}-\frac{1}{r} \frac{n_{z}}{n_{\theta}} \frac{\partial f}{\partial \theta}
\end{aligned}
$$

Les dérivées surlignées suivant $S_{1}$ s'établissent de la même manière soit en conservant les coordonnées $(z, \theta)$ et en éliminant $r$, soit en conservant les coordonnées $(r, \theta)$ et éliminant $z$.

Mais compte tenu des hypothèses simplificatrices $\left(S_{1}\right.$ surface de révolution), il est commode de se placer dans un repère $(u, \theta, v)$ de coordonnées curvilignes orthogonales, $u$ étant pris suivant la méridienne des surfaces $S_{1}, v$ suivant la normale à ces lignes d'écoulement et $\theta$ étant mesuré suivant la direction périphérique.

On peut montrer que les dérivées suivant $S_{1}$ s'expriment par :

$$
\begin{gathered}
\frac{\overline{\partial f}}{\partial u}=\frac{\partial f}{\partial u}-\frac{n_{u}}{n_{v}} \frac{\partial f}{\partial v} \\
\frac{1}{r} \frac{\overline{\partial f}}{\partial \theta}=\frac{1}{r} \frac{\partial f}{\partial \theta}-\frac{n_{\theta}}{n_{v}} \frac{\partial f}{\partial v}
\end{gathered}
$$

mais $n_{u}=n_{\theta}=0$ et $n_{v}=1$, d'où :

$$
\begin{gathered}
\frac{\overline{\partial f}}{\partial u}=\frac{\partial f}{\partial u} \\
\frac{1}{r} \frac{\overline{\partial f}}{\partial \theta}=\frac{1}{r} \frac{\partial f}{\partial \theta}
\end{gathered}
$$

De même, les dérivées suivant $S_{2}$ s'écrivent :

$$
\begin{aligned}
& \frac{\overline{\partial f}}{\partial u}=\frac{\partial f}{\partial u}-\frac{n_{u}}{n_{\theta}} \frac{1}{r} \frac{\partial f}{\partial \theta} \\
& \frac{\partial f}{\partial v}=\frac{\partial f}{\partial v}-\frac{n_{v}}{n_{\theta}} \frac{1}{r} \frac{\partial f}{\partial \theta}
\end{aligned}
$$




\section{Bibliographie}

[1] CAIUS Jacob - Introduction mathématique à la mécanique des fluides.

[2] WU C.M. - A general theory of the three dimensional flow on subsonic and supersonic turbomachines of axial radial and mixed flow types. Transactions of ASME, Nov. 1952.

[3] M VAVRA M. - Aero thermodynamics and flow in turbomachines.

[4] CZIBERE T. - Uber die berechnung der schaufelprofile von strömungs maschinen mit halbaxialer durchstromung. Acta technica Hung, 44/1, 1963.

[5] NYIRI A. - Détermination of the theoretical characteristics of hydraulic machines, based on potential theory. Acta technica Hung, 69/3, 1971.
[6] EREMEEF L.R. - Calcul des écoulements dans les turbomachines en fluide parfait et incompressible. Contrat DGRST $\mathrm{n}^{\circ} 73,7,1765,00,221,75,01$.

[7] EREMEEF L.R. - Method for numerical calculation of velocity distribution for a blade cascade rotating in a perfect incompressible fluid. Periodica Polytechnica Hung, vol. 18, $n^{\circ} 1,(1974)$.

[8] EREMEEF L.R. et PHILIBERT R. - Calculation of flow through turbomachines by an iterative method. Proceedings of the six th conference on fluid machinery, Budapest 1979.

[9] WEGNER M. et LARROZE P.Y. - Comparaison entre écoulements calculés et observés dans des roues Francis. Symposium AIRH, Amsterdam, September 1982.

\section{Discussion}

Président: S. CASACCI

Monsieur BORCIANI entame la discussion en soulevant le problème de l'introduction des pertes dans le calcul. Il demande s'il n'est pas possible de les prendre en compte, filet par filet, à partir de leur expression théorique conformément aux formules données à propos de l'écoulement sur S2.

M. EREMEEF explique qu'il a surtout cherché à obtenir un bon niveau d'énergie entre l'entrée et la sortie de la roue et qu'il a donc pris en compte les pertes de façon globale sans chercher à en avoir une représentation précise dans la roue.

Il souligne l'importance du terme de pertes dans l'équation de l'écoulement méridien, ce terme pouvant être du même ordre de grandeur que le gradient de circulation résiduel dans une turbine et par là-même modifier l'équilibre sous la roue.

M. CASACCI apporte quelques éclaircissements. En fait, dans la méthode quasitridimensionnelle, on ne calcule pas les pertes, on les prend en compte, on les représente par un champ de force à préciser par d'autres voies.

A la suite d'une intervention de M. CARNEVALE, M. EREMEEF confirme les difficultés rencontrées lors de la résolution de l'équation de l'écoulement méridien, quelle que soit la méthode choisie. Dans la méthode intégrale qui est utilisée, les problèmes proviennent des singularités des noyaux.

A la demande de M. LIESS, M. EREMEEF précise les conditions de calcul et les conditions limites imposées. La turbine est divisée en cinq zones: la zone amont, le distributeur, l'espace entre distributeur et roue, la roue mobile et la zone aval.

L'avant distributeur n'est pas pris en compte dans le calcul pas plus que les pertes dans le distributeur. A l'amont du distributeur, l'écoulement est supposé irrotationnel. A l'entrée de la roue, les données sont définies par le distributeur.

En sortie, on impose une vitesse méridienne constante, le plan de sortie étant choisi suffisamment loin en aval pour que la condition limite n'influe pas sur le calcul à l'emplacement du sondage.

M. EREMEEF fait remarquer qu'un changement de conditions limites à l'amont a été essayé mais que cela n'a pas modifié l'écoulement à partir de la sortie du distributeur.
M. VERRY suggère d'imposer la direction de l'écoulement à l'aval plutôt que l'égalité des vitesses méridiennes, cette condition étant moins contraignante.

Sur l'intervention de M. CAZENAVE, M, EREMEEF souligne l'importance de l'avant distributeur dans l'écoulement réel pour limiter les pertes par non-adaptation à l'entrée du distributeur et $M$. CASACCI confirme la nécessité d'une vérification expérimentale des critères choisis pour le calcul du distributeur et la prise en compte des pertes. Il insiste sur la nécessité d'allersretours avec l'expérience du fait de la lourdeur des programmes $\mathrm{S}_{1}-\mathrm{S}_{2}$.

M. MAGRI présente des résultats expérimentaux obtenus sur une turbine FRANCIS analogue à celle présentée. Il apparait que le réseau de lignes de courant se déplace vers la ceinture quand la vitesse de rotation augmente à ouverture constante et que la variation de vitesse de rotation influe sur la distribution des vitesses à l'entrée de la roue.

M. VERRY soulève finalement le problème du couplage entre les surfaces $S_{1}$ et $S_{2}$.

M. EREMEEF rappelle les différents choix de surface $S_{2}$ et les critiques possibles:

- la surface squelette, peu représentative de l'effet grille ; - une surface issue des lignes de courant moyennes des surfaces $\mathrm{S}_{1}$, la détermination de cette surface risquant d'être longue; - une surface "implicite" définie par une loi de circulation (moyenne du champ $\mathrm{RV}_{\theta}$ dans un canal inter-aubes), cette dernière méthode choisie par NEYRPIC, ayant l'avantage de bien représenter les niveaux d'énergie entre l'entrée et la sortie.

M. VERRY observe que dans ce dernier schéma, l'enthalpie totale relative n'est plus conservée sur les lignes de courant, le champ de forces étant normal à la surface squelette et non aux surfaces de glissement $S_{2}$. Il signale que l'équilibre peut être sensiblement modifié dans le cas d'une pompe à aubes très vrillées.

M. VERRY et M. EREMEEF s'accordent à conclure qu'aucune des solutions n'est tout-à-fait satisfaisante et que le problème reste donc ouvert. 


\section{Abstract}

\section{Quasi three dimensional flows in hydraulic turbomachines}

In this paper the three-dimensional flow of a perfect, incompressible fluid through the rotor of a turbomachine is studied with a method using two-dimensional flows:

\section{- meridional flow}

- blade-to-blade flow

We first mention the equations for the three-dimensional case. We then explain the assumptions required to establish the equations of two two-dimensional flows. (In order to be two-dimensional the meridional flow must be axisymetrical).

\section{Meridional flow:}

In order to obtain an evolution of the fluid energy level inside the rotor, it is necessary to introduce a non-conservative field of force $\vec{F}$, normal to $\vec{W}$. The orthogonalit of $\vec{F}$ to $W$ ensures conservation of the relative energy $E_{\text {rel }}$ in the rotor along the path of a particle.

Finally, the meridonal flow equations become :

$$
\begin{gathered}
\frac{\partial\left(\tau_{r} V_{r}\right)}{\partial r}+\frac{\partial\left(\tau_{r} V_{z}\right)}{\partial z}=0 \\
\omega_{\theta}=-\frac{1}{W_{i}} \frac{\partial E_{\mathrm{rel}}}{\partial v}+\frac{1}{r} \frac{W_{\theta}}{W_{i}} \frac{\partial_{r} V_{\theta}}{\partial v} \\
+\frac{n_{v}}{n_{\theta}}\left[\frac{1}{r} \frac{\partial_{r} V_{0}}{\partial u}-\frac{W_{0}}{W^{2}} \frac{\partial E_{\mathrm{rel}}}{\partial u}\right]
\end{gathered}
$$

where $r$ is a blokage factor.

It is to be noted that these equations are written in an orthognal curvilinear system $(v, \theta, u)$.

\section{Blade-to-blade flow.}

The base flow equations on a surface of revolution are made out from the irrotationality of the flow on this surface and from continuity:

$$
\begin{gathered}
\frac{\partial V_{u}}{\partial \theta}-\frac{\partial\left(r V_{\theta}\right)}{\partial u}=0 \\
\frac{\partial\left\lfloor r \Delta b V_{u}\right\rfloor}{\partial u}+\frac{1}{r} \frac{\partial\left\lfloor r \Delta b V_{\theta} \mid\right.}{\partial \theta}=0
\end{gathered}
$$

$\Delta b$ is the thickness of the stream surface.

\section{Iterative resolution technique}

Equation (b) shows that the calculation of meridional flow necessitates knowledge of the distribution of $r V_{\theta}$ over the whole field inside the meridian contour studied.

This distribution is given by the blade-to-blade calculation.

Similarly, the blade cascade calculation requires knowledge of the axisymetrical surface and the fonction $\Delta b$. These data are the result of the meridional calculation.

The iterative process is stopped when two successive flows are deemed to be sufficiently close.

\section{Conclusion}

Figure 4 (or 7 ) gives as example the result of a meridional flow calculation which allows for the presence of the distributeur and the runner.

Probe tests (figure 5,8 ) may be compared to the theorical calculation. Actually good results are obtained with this flow model. 